\title{
ENTIRE FUNCTIONS IN SEVERAL VARIABLES WITH CONSTANT ABSOLUTE VALUES ON A CIRCULAR UNIQUENESS SET ${ }^{1}$
}

\author{
S. BOCHNER
}

If for a function $\phi(z)=\sum_{0}^{\infty} a_{p} z^{p}$ in the entire $z$-plane we have $|\phi(z)|=1$ on $|z|=1$, then the product

$$
\sum_{0}^{\infty} a_{p} z^{p} \cdot \sum_{0}^{\infty} \frac{\bar{a}_{q}}{z^{q}}
$$

which is analytic in $z \neq 0$ has value 1 on $|z|=1$. Therefore it is identically 1 , and thus $\phi(z) \neq 0$ for $z \neq 0$. Therefore $\phi(z)=z^{p} h(z)$ where $h(z) \neq 0$ everywhere. But again $|h(z)|=1$ on $|z|=1$, and for such an $h(z)$ we have $h(z)=c$, so that $\phi(z)=c z^{p}$. By the use of the same method, Bojanic and Stoll [1] have recently given the following generalization to functions which are holomorphic in the entire $\mathbf{C}^{n}$, for any $\boldsymbol{n}$.

THEOREM 1. If for an entire function $f(z) \equiv f\left(z_{1}, \cdots, z_{n}\right)$ we have

$$
\left|f\left(\zeta_{1}, \cdots, \zeta_{n}\right)\right|=1
$$

on the set

$$
\left|\zeta_{1}\right|=1, \cdots,\left|\zeta_{n}\right|=1
$$

then

$$
f\left(z_{1}, \cdots, z_{n}\right)=c z_{1}^{p_{1}} \cdots z_{n}^{p_{n}} .
$$

The authors invoke the lemma that if an entire function is $\neq 0$ for $z_{1} \cdots z_{n} \neq 0$ then it can be represented as a product

$$
c z_{1}^{p_{1}} \cdots z_{n}^{p_{n}} h(z)
$$

in which $h(z) \neq 0$ everywhere. We propose to avoid taking recourse to this lemma and to obtain a more systematic theorem in the process.

We replace the point set (1) by a general point set $S$ having the following properties

(i) $S$ is circular, that is, if $\left(\zeta_{1}, \cdots, \zeta_{n}\right) \in S$, then also $\left(\zeta_{1} t, \cdots, \zeta_{n} t\right)$ $\in S$ for any $|t|=1$.

(ii) $S$ is connected.

(iii) $S$ is a uniqueness set for entire functions, in the sense that if

Received by the editors February 27, 1961.

1 This research was supported by the United States Air Force through the Office of Scientific Research of the Air Force Research and Development Command. 
$f(z)$ is 0 on $S$ it is $\equiv 0$. We note that $S$ is of this kind if it is part of the boundary of a domain $R$ such that, for $z$ in $R, f(z)$ can be represented by a suitable Cauchy integral in which integration extends over $S$ only.

THEOREM 2. If for an entire function we have $|f(\zeta)|=1$ on a set $S$ then $f(z)$ is a homogeneous polynomial

$$
A\left(z_{1}, \cdots, z_{n}\right)=\sum_{p_{1}+\cdots+p_{n}=p} a_{p_{1} \cdots p_{n}} z_{1}^{p_{1}} \cdots z_{n}^{p_{n}}
$$

of some finite degree $p \geqq 0$.

Proof. For any fixed $(\zeta) \in S$ we form the function

$$
\phi(z)=f\left(\zeta_{1} z, \cdots, \zeta_{n} z\right)
$$

in C1. By property (i) of $S$ we have $|\phi(z)|=1$ on $|z|=1$, and hence by our introductory statement we have $\phi(z)=c z^{p}$, that is

$$
f\left(\zeta_{1} z, \cdots, \zeta_{n} z\right)=z^{p} f\left(\zeta_{1}, \cdots, \zeta_{n}\right) .
$$

Up to here the exponent $p$ is a function of $\zeta$. However it follows from

$$
|z|^{p}=\left|f\left(\zeta_{1} z, \cdots, \zeta_{n} z\right)\right|
$$

(for $z=2$, say) that $p$ is a continuous function on $S$. Furthermore it is integer-valued, and $S$ is connected by property (ii). Therefore $p$ is a constant number.

We can now form the difference

$$
f\left(w_{1} z, \cdots, w_{n} z\right)-z^{p} f\left(w_{1}, \cdots, w_{n}\right) .
$$

It is an entire function in $w_{1}, \cdots, w_{n}$ and $z$, and it is 0 for $(w) \in S$. By property (iii) it is $\equiv 0$. If we now introduce the power series for $f(z)$, the conclusion of Theorem 2 follows.

We next add one further property of $S$ which is much more structural than the preceding ones.

(iv) On $S$ we have

$$
\xi_{\nu}=\frac{\lambda_{\nu}(\zeta)}{D(\zeta)}, \quad \nu=1, \cdots, n,
$$

where $\lambda_{1}(z), \cdots, \lambda_{n}(z), D(z)$ are (homogeneous) polynomials in $z_{1}, \cdots, z_{n}$.

If now we introduce the factorization into irreducible polynomials

$$
D(z)=D_{1}(z)^{q_{1}} \cdots D_{m}(z)^{q_{m}}
$$

then the following conclusion results. 
THEOREM 3. Furthermore, we have

$$
f\left(z_{1}, \cdots, z_{n}\right)=c D_{1}(z)^{p_{1}} \cdots D_{m}(z)^{p_{m}}
$$

for some exponents $p_{1} \geqq 0, \cdots, p_{m} \geqq 0$.

Proof. If with the coefficients of (2) we form the polynomial

$$
B\left(z_{1}, \cdots, z_{n}\right)=\sum_{p_{1}+\cdots+p_{n}=p} \overline{a_{p_{1}} \cdots p_{n}} \lambda_{1}^{p_{1}} \cdots \lambda_{n}^{p_{n}}
$$

then our assumption

$$
|f(\zeta)|^{2}=1=f(\zeta) \overline{f(\zeta)}
$$

implies that we have

$$
A(z) \cdot B(z)=D(z)^{p}
$$

on $S$. Using again (iii) we conclude that this holds identically in $z$. But all factors in (5) are polynomials and therefore (5) implies (4) by simple algebra.

REMARK. If also $\left|D_{\nu}(\zeta)\right|=1, \nu=1, \cdots, n$, then conversely every function (4) has constant absolute value on $S$.

Theorem 1 subsumes under Theorem 3 if we put

$$
D(z)=z_{1} \cdots z_{n} ; \quad \lambda_{\nu}(z)=\frac{D(z)}{z_{\nu}}, \quad \nu=1, \cdots, n .
$$

But we also obtain interesting statements for some types of symmetric domains. Assume for instance that $n=k^{2}$ and that our variables $z_{1}, \cdots, z_{n}$ constitute a square array $\left\{z_{p q}\right\}, p, q=1, \cdots, k$. The associated "natural" uniqueness set is formed by the unitary matrices

$$
\sum_{r=1}^{k} \zeta_{p r} \zeta_{q r}=\delta_{p q}
$$

see [2].

This gives

$$
\xi_{\mu \nu}=\frac{\lambda_{\mu \nu}(\zeta)}{\operatorname{det}|\zeta|}
$$

where $\lambda_{\mu \nu}$ are certain minors of the matrix $\left\{\zeta_{p q}\right\}$ and

$$
D(\zeta)=\operatorname{det}\left|\zeta_{p q}\right|
$$


is its determinant. Now, the determinant is an irreducible polynomial and $|D(\zeta)|=1$. Hence the following theorem.

THEOREM 4. If $f\left(z_{r s}\right)$ is defined holomorphic over the entire matrix space, then it has absolute value 1 on the unitary set (6) if and only if

$$
f(z)=e^{i \alpha}\left(\operatorname{det}\left|z_{r s}\right|\right)^{p}
$$

for some integer $p \geqq 0$.

\section{BIBLIOGRAPHY}

1. R. Bojanic and W. Stoll, A characterization of monomials, Proc. Amer. Math. Soc. this issue, [pp. 115-116].

2. S. Bochner, Group invariance of Cauchy's formula in several variables, Ann. of Math. (2) 45 (1944), 686-707.

Princeton University

\section{ON A CRITERION FOR DETERMINATE MOMENT SEQUENCES}

DAVID S. GREENSTEIN

On page 20 of [4], the following criterion is given as sufficient for the determinacy of a Hamburger moment sequence $\left\{\mu_{n}\right\}$ :

$$
\lim \inf \left(\mu_{2 n}^{1 / 2 n} / n^{2}\right)<\infty .
$$

Attributed to Perron [2], it is obtainable only by transforming a criterion for Stieltjes determinacy due to Perron. In doing so, I find (1) not to follow from Perron's result. In this note, I shall make the proper correction to eliminate confusion caused by the error (e.g., (1) if valid would be more general than Carleman's well known criterion [1]). I also give an example to show that (1) is invalid.

Symmetrization of all mass distributions with the moments $\mu_{n}$ shows that $\left\{\mu_{n}\right\}$ is determinate provided that there is no more than one symmetric distribution with the moments $\mu_{0}, 0, \mu_{2}, 0, \cdots$ But the latter condition is easily shown to be equivalent to Stieltjes determinacy of the moment sequence $\left\{\mu_{2 n}\right\}$ (not the same as Hamburger determinacy of $\left.\left\{\mu_{2 n}\right\}[4]\right)$.

Perron [2] gives as a sufficient condition for Stieltjes determinacy of $\left\{\mu_{2 n}\right\}$

Received by the editors February 14, 1961. 